\title{
Role of Thick-Lithium Fluoride Layer in Energy Level Alignment at Organic/Metal Interface: Unifying Effect on High Metallic Work Functions
}

\author{
Zhengyi Sun, Shengwei Shi, Qinye Bao, Xianjie Liu and Mats Fahlman
}

\section{Linköping University Post Print}

\section{Tweet}

N.B.: When citing this work, cite the original article.

Original Publication:

Zhengyi Sun, Shengwei Shi, Qinye Bao, Xianjie Liu and Mats Fahlman, Role of Thick-Lithium Fluoride Layer in Energy Level Alignment at Organic/Metal Interface: Unifying Effect on High Metallic Work Functions, 2015, ADVANCED MATERIALS INTERFACES, (2), 4, 1400527. http://dx.doi.org/10.1002/admi.201400527

Copyright: Wiley: 12 months http://eu.wiley.com/WileyCDA/

Postprint available at: Linköping University Electronic Press http://urn.kb.se/resolve?urn=urn:nbn:se:liu:diva-117235 


\title{
WILEY-VCH
}

\section{Full Paper}

Role of thick lithium fluoride layer in energy level alignment at organic/metal interface: Unifying effect on high metallic work functions

Zhengyi Sun, * Shengwei Shi, Qinye Bao, Xianjie Liu and Mats Fahlman*

Dr. Z. Sun, Dr. S. Shi, Q. Bao, Dr. X. Liu, Prof. M. Fahlman

Department of Physics, Chemistry and Biology (IFM), Linköping University, SE-581 83 Linköping, Sweden

E-mail: zhsun@ifm.liu.se; mats.fahlman@liu.se

Keywords: energy level alignment; organic/metal interface; thick lithium fluoride; ICT model.

\begin{abstract}
We have investigated the function of $\sim 3 \mathrm{~nm}$ thick lithium fluoride (LiF) buffer layers in combination with high work function metal contacts such as coinage metals and ferromagnetic metals for use in organic electronics and spintronics. The energy level alignment at organic/LiF/metal interfaces is systematically studied using photoelectron spectroscopy and the integer charge transfer model. The thick-LiF buffer layer is found to pin the Fermi level to $\sim 3.8 \mathrm{eV}$ regardless of the work function of the initial metal due do energy level bending in the LiF layer caused by depletion of defect states. At $3 \mathrm{~nm}$ thickness, the LiF buffer layer provides full coverage, and the organic semiconductor adlayers are found to physisorb with the consequence that the energy level alignment at the organic/LiF interface follows the integer charge transfer model's predictions.
\end{abstract}




\section{WILEY-VCH}

\section{Introduction}

Electron transport at metal-molecule interface significantly affects the performance of electronic devices based on organic semiconductors (OSC). To get efficient electron transport i.e. electron injected into or extracted from the lowest occupied molecular orbit (LUMO) of organic material, a low work function (WF) electrode, such as alkali or alkaline-earth metals, is generally required. However, low WF metals are chemically reactive and easily oxidize in atmosphere, which influences the electrode stability. Thus, there is a conflict between device performance and lifetime, and as a result, device fabrication has to be carried out in an inert atmosphere and encapsulation is needed for device operation, which increases both the cost and complexity of production.

Buffer layers play an essential role in organic electronics, since they solve this problem by the interfacial modification effect. Lithium fluoride $(\mathrm{LiF})$ is a typical insulating material that is widely used as buffer layer at organic/metal interfaces to improve electron injection and hence device performance in organic electronics and spintronics, such as organic lightemitting diode (OLED), organic photovoltaics (OPV), organic thin-film transistor (OTFT) and organic spin valves (OSV). ${ }^{[1-6]}$ Initially, the insertion of an ultrathin LiF layer between the organic film and $\mathrm{Al}$ cathode was found to significantly improve the performance of $\mathrm{Alq}_{3^{-}}$ based OLEDs, ${ }^{[1]}$ for which the typically LiF thickness is below $1 \mathrm{~nm}$. Here the enhancement has been attributed to the reduction of electron-injection-barrier (EIB) due to chemical interaction (doping), ${ }^{[7]}$ though some other mechanisms, including tunneling, ${ }^{[1]}$ have been proposed as well. ${ }^{[8-11]}$ However, for many other metals, especially those with high WF, LiF layer of such thickness range $(<1 \mathrm{~nm})$ does not work well. ${ }^{[12]}$ Recently, it has been found that for OLEDs with high WF metals as cathodes, such as the noble metals Ag and Au as well as non-noble metal $\mathrm{Cu}$ (together called coinage metals), a thicker LiF buffer layer can however significantly improve the electron injection and thus the device performance. ${ }^{[13-15]}$ Intriguingly, the optimal thickness of LiF buffer layer is nearly the same for the three coinage 


\section{WILEY-VCH}

metal cathodes: $\sim 3 \mathrm{~nm}$. Obviously, the role of $\mathrm{LiF}$ buffer layer with such thick range is different from the ultrathin case due to the inert chemical character of noble metals and the sharp-cut interface (absent of metal penetration and doping into organic layer). ${ }^{[16]}$ The enhancement effect is speculated to arise from EIB reduction through enhanced tunneling. ${ }^{[13-}$ ${ }^{18]}$. Nevertheless, the tunneling model is based on the traditional assumption of a common vacuum level that is not necessarily true, as a charge transfer potential step can occur if the Fermi level position of the LiF-modified metal contact induces either oxidation or reduction of the organic semiconductor layer. ${ }^{[19,20]}$ The direct evidence of interfacial energy level alignment for the thick-LiF case is still lacking and the quantitative measurements are thus required to elucidate the injection enhancement mechanism.

In the hybrid organic spintronic devices, spin injection, spin transport and spin extraction are three key processes. Among them, both the first and the last steps take place at OSC/FMelectrode interface, also called spinterface. ${ }^{[21]}$ Therefore the proper design of the spinterface is crucial for device performance. ${ }^{[22,23]}$ The interface energetics is one of the dominant factors governing the efficacy of a spinterface since the optimized energy level match will facilitate the carrier injection/extraction thus the spin injection/extraction. LiF is also utilized as buffer layer in OSV with the ferromagnetic (FM) electrode to enhance device performance and make it more stable, or even to control the polarization of extracted spins and the sign of magnetoresistance. ${ }^{[24,25]}$ In so-called spin-OLEDs, ${ }^{[26]}$ efficient electroluminescence demands carrier-injection balance, which requires a good electron-injection from FM-cathode (electron is typically the minority carrier in general OLED and OSV structures). Due to the high WF of FM metals, the incorporation of LiF interlayer is then also necessary for OLEDs with the FMcathode. Therefore, it is worth studying the energetics of thick-LiF on FM surfaces. For the sake of convenience, throughout the article, thick-LiF refers to the $\mathrm{LiF}$ layer with the thickness around $3 \mathrm{~nm}$ while ultrathin refers to the conventional thickness below $1 \mathrm{~nm}$. 


\section{WILEY-VCH}

Ultraviolet photoelectron spectroscopy (UPS) is an experimental technique to measure directly the molecular occupied energy levels in the valence band region and being particularly surface sensitive, is very suitable to check energetics at an interface. The WF can be obtained from the measured energy of the secondary electron cut-off $\left(\mathrm{E}_{\mathrm{co}}\right)$ and the photon energy $(\mathrm{h} v)$ from the relation $\mathrm{WF}=\mathrm{h} v-\mathrm{E}_{\mathrm{co} .}{ }^{[27]}$ The so-called secondary electrons are photoemitted electrons that have undergone inelastic scattering producing an energy spectrum background superimposed onto the features generated from the so-called primary (elastically scattered) photoemitted electrons. The $\mathrm{E}_{\mathrm{co}}$ represents the photoemitted electrons just escaping the surface with $0 \mathrm{eV}$ kinetic energy, and the difference between $\mathrm{h} v$ and $\mathrm{E}_{\mathrm{co}}$ must then be the work function.

The change in WF, often referred to as $\Delta$, can be monitored by re-measuring the $\mathrm{E}_{\mathrm{co}}$ after a deposition step has been carried out (LiF or in situ condensation of a molecular (sub) monolayer, etc.). Hence, the relation between energy levels at a heterojunction can be measured, both in reference to the vacuum level and to the Fermi level.

In this study, we apply the UPS technique to investigate the energy level alignment at metal-organic interface with $\sim 3 \mathrm{~nm}$ thick-LiF interlayer and to derive the effective mechanism for the EIB reduction at such thickness range. The so-called coinage metals (Au, $\mathrm{Ag}$ and $\mathrm{Cu}$ ), which have been verified to be the efficient cathode of OLED matching with 3 $\mathrm{nm} \mathrm{LiF}$ buffer layer in previous work, were chosen as the metal substrates. It is found that the $3 \mathrm{~nm}$ LiF interlayer will not only reduce the highest occupied molecular orbit (HOMO) of the $\mathrm{Alq}_{3}$ overlayer but also tune it to the same position for all the coinage metals. Then the metal substrates are extended to the FM metals (Fe, Co and Ni), combining with organic molecules $\mathrm{Alq}_{3}$ and $\mathrm{C}_{60}$, as the two molecules are good candidates for spacer layer in OSVs. ${ }^{[28-30]}$ Unlike the case of ultrathin LiF, thick-LiF yields a common WF for all of the metals studied and consequently the same EIBs for the respective OSC (also unlike the ultrathin LiF case). The 


\section{WILEY-VCH}

specific interfaces studied in this work enable us to formulate a general description of the energetics at organic/metal interfaces with thick-LiF layer.

\section{Experimental Results}

\subsection{Valence Band of Alqu on Coinage Metals}

Firstly, the electronic structure of $\mathrm{Alq}_{3}$ deposited on $\mathrm{Au}, \mathrm{Ag}$ and $\mathrm{Cu}$ with and without $\sim 3$ $\mathrm{nm} \mathrm{LiF}$ interlayer has been investigated to study the role of thick-LiF in OLEDs with coinage metal cathodes. The results are shown in Figure 1. The thickness of $\mathrm{Alq}_{3}$ increases from bottom to top as indicated by long arrow in Figure 1a until the substrate is fully covered subject to the curve showing the typical bulk $\mathrm{Alq}_{3}$ spectra. The HOMO level position is defined as the leading edge of highest molecular orbital peak that is derived from the cross of the dash lines shown in Figure 1a. When $\mathrm{Alq}_{3}$ is adsorbed on bare coinage metal substrates, the HOMO level of the bulk $\mathrm{Alq}_{3}$ layer are located at the binding energy of $1.7 \mathrm{eV}, 2.1 \mathrm{eV}$ and $2.0 \mathrm{eV}$ for $\mathrm{Au}, \mathrm{Ag}$ and $\mathrm{Cu}$ substrate, respectively. The result is corresponding to the WFs of $\mathrm{Au}, \mathrm{Ag}$ and $\mathrm{Cu}(5.3 \mathrm{eV}, 4.5 \mathrm{eV}$ and $4.7 \mathrm{eV}$, respectively, derived from the secondary electron cut-off in Figure 4 that is reproducible in our experiments). With the increase of $\mathrm{Alq}_{3}$ thickness, the HOMO peak shifts slightly and monotonically to the higher binding energy, which is a typical phenomenon in UPS measurements of $\mathrm{Alq}_{3}$ evaporated in vacuum ${ }^{[31]}$ and believed to be related to the surface potential. ${ }^{[32]}$ This is also the reason we cease the $\mathrm{Alq}_{3}$ deposition as soon as the UPS curve shows typical bulk characteristic to avoid the influence of such effect. When $\sim 3 \mathrm{~nm} \mathrm{LiF}$ layer is deposited on Au substrate, the HOMO level of bulk $\mathrm{Alq}_{3}$ subsequently deposited shifts to the binding energy of $\sim 2.5 \mathrm{eV}$. The results show a significant descent of HOMO level versus Fermi level with the introduction of thick-LiF interlayer, by as large as $0.8 \mathrm{eV}$. It also suggests a same value reduction of EIB, which is regarded as the energy difference between the cathode Fermi level and Alq ${ }_{3}$ LUMO, since the optical band gap between HOMO and LUMO should be invariant $\left(2.8 \mathrm{eV}\right.$ for $\mathrm{Alq}_{3}$ in 


\section{WILEY-VCH}

common use is $\operatorname{chosen}^{[33]}$ ). For $\mathrm{Ag}$ and $\mathrm{Cu}$ substrate, analogous results are obtained. The HOMO level of bulk $\mathrm{Alq}_{3}$ deposited on the substrate with $3 \mathrm{~nm} \mathrm{LiF}$ interlayer shifts to $2.5 \mathrm{eV}$ relative to Fermi level as well, for both $\mathrm{Ag}$ and $\mathrm{Cu}$ substrates, showing a descent of HOMO by $0.4 \mathrm{eV}$ and $0.5 \mathrm{eV}$, respectively. Such effect induces electron injection enhancement for all the coinage metal cathodes and thus improves the performance of corresponding OLEDs. The same experiment also carried out on the Au substrate covered by ultrathin $\mathrm{LiF}(\sim 0.8 \mathrm{~nm})$, the spectra shift trend versus thickness increase of $\mathrm{Alq}_{3}$ is similar to that on the bare Au substrate (see Figure S1 in supporting information), which is probably ascribed to the incomplete coverage of ultrathin $\mathrm{LiF}$ on the amorphous substrate. The HOMO level of $\mathrm{Alq}_{3}$ at $\mathrm{Alq}_{3} /$ ultrathin $\mathrm{LiF}(0.8 \mathrm{~nm}) / \mathrm{Au}$ interface is located at $\sim 2.1 \mathrm{eV}$ versus Fermi level, which means ultrathin-LiF could also reduce the EIB to some extent but not as much as thick-LiF does, especially for the high WF cathode like Au. Therefore, for the ultrathin LiF, the supposed-existing chemical reaction takes dominant effect, which induces the gap state and normally occurs in the typical case of $\mathrm{Al}$ cathodes only. ${ }^{[34]}$ Finally, it is noticeable that the HOMO level of $\mathrm{Alq}_{3}$ moves to the same position of $2.5( \pm 0.05) \mathrm{eV}$ versus Fermi level, for all the three coinage metals, despite of the different WFs. This phenomenon, which will be discussed later in detail, could account for why the optimal thickness of LiF buffer layer is all the same for $\mathrm{Au}, \mathrm{Ag}$ and $\mathrm{Cu}$ cathodes. ${ }^{[13-15]}$

\subsection{Energy Level Alignment of Alq3 on Ferromagnetic Metals}

Figure 2a shows UPS spectra of $\mathrm{Alq}_{3}$ adsorbed on Fe substrate with and without a $3 \mathrm{~nm}$ $\mathrm{LiF}$ interlayer. From the secondary electron cut-off, the WF of Fe substrate is $4.6 \mathrm{eV}$, and it turns to $3.5 \mathrm{eV}$ after the deposition of $\mathrm{Alq}_{3}$, which means an interface dipole as large as $1.1 \mathrm{eV}$ is formed at the interface. The HOMO level of $\mathrm{Alq}_{3}$ is located at the binding energy of $2.3 \mathrm{eV}$ below Fermi level. The ionization potential (IP) of $\mathrm{Alq}_{3}$ can thus be deduced to be $5.8 \mathrm{eV}$, the typical value reported in former literatures. ${ }^{[33,35,36]}$ When $3 \mathrm{~nm} \mathrm{LiF}$ interlayer is deposited on 


\section{WILEY-VCH}

$\mathrm{Fe}$, there is an interface dipole of $0.9 \mathrm{eV}$ at the interface. In the valence band region, there is a broad peak at the binding energy of $8-13 \mathrm{eV}$ which is derived from F2p features. ${ }^{[37]}$ The Alq 3 deposition on $\mathrm{LiF}$ further induces another interface dipole $(0.4 \sim 0.5 \mathrm{eV})$ and the HOMO level position shifts to $2.5 \mathrm{eV}$. The right side of Figure $2 \mathrm{a}$ shows the energy level alignment diagrams deduced from the UPS spectra, in which the cases of $\mathrm{Alq}_{3} / \mathrm{Fe}$ interface and $\mathrm{Alq}_{3} /$ thick-LiF/Fe interface are juxtaposed to make the easier comparison. The typical optical band gap of $2.8 \mathrm{eV}$ is used to get LUMO level position. ${ }^{[33]}$

For Co and Ni substrate, quite similar results are obtained. The UPS spectra can be found in the supporting information (Figure $2 \mathrm{~S}$ ) and the energy level alignment diagrams derived are shown in Figure 2b. The interface dipole at $\mathrm{Alq}_{3} / \mathrm{Co}$ interface is $1.4 \mathrm{eV}$ and the HOMO level is located at $2.1 \mathrm{eV}$, exactly the same as the previous work despite of the different deposition sequence. ${ }^{[38]}$ When a $3 \mathrm{~nm} \mathrm{LiF}$ interlayer is inserted, the total interface dipoles change to 1.7 $\mathrm{eV}$ while the HOMO level shifts to the binding energy of $2.5 \mathrm{eV}$ versus Fermi level. For Ni substrate, the interface dipole at $\mathrm{Alq}_{3} / \mathrm{Ni}$ interface is $1.1 \mathrm{eV}$ and the $\mathrm{HOMO}$ level is located at $1.9 \mathrm{eV}$, while the two values change to $1.5 \mathrm{eV}$ and $2.5 \mathrm{eV}$, respectively. If we combine the results of FM metals with the previous results of coinage metals, an interesting phenomenon is found. For all the metals used by now, the extra $3 \mathrm{~nm} \mathrm{LiF}$ interlayer pins the HOMO level of the subsequently deposited $\mathrm{Alq}_{3}$ to the same position of $\sim 2.5 \mathrm{eV}$ below the Fermi level. Accordingly, all the LUMO level positions should be the same as well. It is inferred that the barriers of electron injection, including the extra barrier induced by the LiF layer, are the same for the metals with different WFs when the thick-LiF interlayer exists. That is to say, the thick-LiF has a sort of unifying effect on the metals with different WFs. Furthermore, the effective WF of thick-LiF-coated metals are $\sim 3.8 \mathrm{eV}$ and the effective WF of $\mathrm{Alq}_{3}$-coated metals with thick-LiF interlayer are tuned to the same value of $3.3 \mathrm{eV}( \pm 0.1 \mathrm{eV})$ !

\subsection{Energy Level Alignment of C60 on Different Work Function Metals}




\section{WILEY-VCH}

In subsequent experiment, we will discuss on $\mathrm{C} 60$ in the same system as previous $\mathrm{Alq}_{3}$ case. The energy level alignments at C60/FM metal and C60/thick-LiF/FM metal interfaces deduced from the UPS spectra are as shown in Figure 3 (the UPS spectra for Fe and Ni cases are shown in Figure 3S). The WFs of $\mathrm{Fe}$, $\mathrm{Co}$ and $\mathrm{Ni}$ in the experiment are $4.6 \mathrm{eV}, 5.0 \mathrm{eV}$ and $4.9 \mathrm{eV}$, respectively. Different from the $\mathrm{Alq}_{3}$ case, there is no large interface dipole formed when C60 is directly deposited onto the FM metals. The HOMO levels are located at the binding energy of $1.5 \mathrm{eV}, 1.6 \mathrm{eV}$ and $1.4 \mathrm{eV}$ versus Fermi level for the $\mathrm{Co}, \mathrm{Fe}$ and $\mathrm{Ni}$ substrates, respectively. The IP of $\mathrm{C} 60$ is measured as $6.4 \mathrm{eV}( \pm 0.1 \mathrm{eV})$, corresponding to the typical value in previous work. ${ }^{[39]}$ We assume a band gap of solid C60 of $2.3 \mathrm{eV}$ as determined by PES and IPES techniques ${ }^{[40]}$ (there is also a similar value of $2.6 \mathrm{eV}$ in another experiment $^{[41]}$ ), and then the LUMO level position can be assigned as shown in Figure 3. The barrier is relatively high $(0.8 \pm 0.1 \mathrm{eV})$ and not favorable for the electron injection. Similar to the effect on $\mathrm{Alq}_{3}$, the introduction of $3 \mathrm{~nm} \mathrm{LiF}$ interlayer shifts the HOMO level of $\mathrm{C} 60$ and pins it to the same position of $2.2 \mathrm{eV}$ for all three FM metals, see Figure 3. Consequently the effective WFs of C60 coated FM metals are tuned to the same value of $4.3 \mathrm{eV}( \pm 0.1 \mathrm{eV})$ ! The LUMO level hence is located quite close to the Fermi level ( $0.1 \mathrm{eV}$ higher or equal), implying a significantly reduced EIB.

Since the thick-LiF interlayer shows the same effect on FM metals together with C60 adlayers, in order to further verify such phenomena in other high WF electrodes case, the coinage metals are investigated as well. Figure 4 shows the UPS spectra at the interfaces of C60/coinage metals with the thick-LiF interlayer. It can been seen that the HOMO levels of $\mathrm{C} 60$ on $3 \mathrm{~nm} \mathrm{LiF}$ interlayer are pinned to $2.1 \sim 2.2 \mathrm{eV}$ for three coinage metals $(\mathrm{Au}, \mathrm{Ag}$ and $\mathrm{Cu}$ ) and the WFs of C60 are the same value of $4.3 \mathrm{eV}$ as well. All the values are the same as FM metal cases within the measurement uncertainty. Therefore, the capability of thick-LiF to provide a common WF for the high WF metals studied is further confirmed and thus likely to be general to other molecules and metals. 


\section{WILEY-VCH}

\section{Discussions}

There is a thickness fluctuation around $3 \mathrm{~nm}$ of the $\mathrm{LiF}$ interlayer in our experiments $(2.7 \sim 3.4 \mathrm{~nm})$. It is noticeable that such fluctuation has little influence on the final energy level alignment. According to calculations in Prada et al.'s work, ${ }^{[42]}$ the effective WF of metal substrate is lowered by just a single intact layer of LiF and does not change upon increasing LiF thickness. However, in an actual experiment by Watkins and co-workers, the effective WF always drops continuously until LiF thickness increases to $2 \mathrm{~nm} .{ }^{[43]}$ This suggests that LiF does not form an intact layer when the thickness is thin (less than $2 \mathrm{~nm}$ ), especially on the metal support with amorphous surface. There is also the issue of LiF interaction with the substrate, but even for highly reactive metals such as aluminum, LiF does not dissociate upon deposition. ${ }^{[44-46]}$ Still, for reactive metals such as Co and Ni, the initial LiF layer at the surface may undergo some chemical modification necessitating thicker films than a monolayer to achieve an intact LiF layer. It can thus be inferred that for nanoscale thick-LiF layers on a non-atomic-flat substrate (even though full coverage), the lattice defect is inevitable. Our UPS results support the existence of lattice defect in $\mathrm{LiF}$ layer as well. Besides the main peak from F2p there is also a weak but definite tail structure at the binding energy of $5-8 \mathrm{eV}$ in the UPS spectrum of thick-LiF. Such structure can be ascribed to the lattice defects of $\mathrm{LiF}^{[47]}$ It is flat and undistinguishable in Figure 2a and Figure 3a due to the very high adjacent main peak but can be easily seen in Figure 1. Thus, the defect states will exist and enable the charge transfer and the pinning of Fermi level. Besides, according to the theoretical calculation, ${ }^{[42]}$ metalinduced gap states of $\mathrm{LiF}$ at the $\mathrm{LiF} /$ metal interface are present, which also probably contribute to the energy level pinning. Figure 5a shows a LiF-assisted energy level pinning regime that we propose to explain our experiment results at the organic/thick- $\mathrm{LiF} / \mathrm{metal}$ interfaces. As indicated in the dotted box, the presence of the thick-LiF makes a "new surface". Due to the Fermi level pinning, such surface is characterized by a pinning of the 


\section{WILEY-VCH}

Fermi level independent of the metal WF. We propose that this pinning is caused by energy level bending through depletion of defect states in the LiF film. Therefore, with the energylevel-bending-derived effect of thick-LiF, the thick-LiF/metal combination can be regarded as a "new" substrate with a well-defined WF of $\sim 3.8 \mathrm{eV}$. Organic molecules subsequently adsorbed on this surface will undergo energy level alignment in reference to this Fermi level position, not the original metal's.

The new surface is considered as inert due to the full coverage of LiF, so the energy level alignment will follow the so-called integer charge transfer (ICT) model. ${ }^{[20,27,48]}$ For the case of an $\mathrm{Alq}_{3}$ overlayer and a starting $\mathrm{WF}$ of the surface of $\sim 3.8 \mathrm{eV}$, a resulting Fermi level at 3.3 3.4 eV is predicted by the ICT model, ${ }^{[31]}$ see Figure $5 b$, in excellent agreement with our measurements. For the case of a C60 overlayer and a starting WF of the surface of $\sim 3.8 \mathrm{eV}$, the creation of so-called ICT states is expected through reduction of C60 molecules at the interface, which in turn is expected to result in a Fermi level pinned to the $E_{\mathrm{ICT}-}$ energy, which for C60 films is $\sim 4.4 \mathrm{eV},{ }^{[49]}$ see Figure $5 \mathrm{~b}$, again in line with the results from our measurements.

Here we stress again that the so-called thick just refers to being relatively thicker than the conventionally used ultrathin thickness $(<1 \mathrm{~nm})$, but still in the range of nanoscale $(\sim 3 \mathrm{~nm})$. On one hand, the LiF layer needs to be thick enough to form the full coverage so as to obtain the unified energy level and pacify the metal substrate (preventing covalent bonding with the subsequent OSC layer). On the other hand, it cannot be too thick since then resistance losses from moving charge across the $\mathrm{LiF}$ layer will outweigh the gain from the improved energy level alignment with the OSC. The presence of the thick-LiF interlayer at the organic/metal interface could counteract the impact of the different metallic WF from the view of energetics and thus extend the selection of electrode materials to functional metals with high WF. Moreover, it makes the ICT model applicable so that the energy level alignment at the 


\section{WILEY-VCH}

organic/thick-LiF/metal interface can be predicted, which is helpful for the design of organic electronic and spintronic devices.

\section{Conclusion}

By investigating the energy level alignment at the organic/metal interfaces with and without $\sim 3 \mathrm{~nm} \mathrm{LiF}$ interlayer, we have demonstrated the mechanism of thick-LiF as the interfacial buffer layer between the organic materials and the metals. Different from the ultrathin $\mathrm{LiF}$ layer, the electron-injection enhancement of thick-LiF arises from the energy level bending in the LiF layer due to depletion of defect states in the gap. The energy level bending makes a "new surface" characterized by a Fermi level $(\sim 3.8 \mathrm{eV})$, which is independent of the original metallic WF. Moreover, the introduction of thick-LiF interlayer renders the surface inert, and organic material subsequently deposited on the thick-LiF/metal composite substrate is thus physisorbed and the energy level alignment at this interface then follows the ICT model. Our work suggests a generality of the thick-LiF buffer layer effect that improves the electron-injection for the metallic functional materials with high WF, and that the energy level alignment for such systems can be predicted with a $\pm \sim 0.1 \mathrm{eV}$ accuracy by the ICT model.

\section{Experimental Section}

All the experiments were carried out in the ultra-high vacuum (UHV) system which comprised a preparation chamber and an analysis chamber with the base pressure of $1 \times 10^{-9}$ and $3 \times 10^{-10}$ mbar, respectively. The coinage metals were prepared by deposition on silicon wafer previously with a thickness of $\sim 300 \mathrm{~nm}$ and, when we did the experiment, the substrate was in-situ cleaned by argon sputtering in the preparation chamber to get fresh surface. FM metals were deposited on Au-coated silicon wafer in situ using e-beam evaporator. Thus, all metal substrates are atomically clean. The organic molecules ( $\mathrm{Alq}_{3}$ and $\left.\mathrm{C} 60\right)$ and $\mathrm{LiF}$ layer were evaporated from a simple home-made Knudsen cell and the thickness was determined 


\section{WILEY-VCH}

from the attenuation of the core-level signals of the underlying substrate. After each deposition, the sample was transferred from preparation chamber to analysis chamber without breaking UHV and the UPS measurement was performed there. A negative bias $(-3 \mathrm{~V})$ is applied on the sample to get a clear cut-off. We use HeI light where hv $=21.2 \mathrm{eV}$. The measurement sequence involved the characterization of the bare metal substrates (and $\mathrm{LiF}$ layer for the with-LiF case) and the subsequent stepwise deposition and characterization of molecular add-layers.

\section{Supporting Information}

Supporting Information is available from the Wiley Online Library or from the author.

\section{Acknowledgements}

This research is financially supported by the European Commission FP7 collaborative project HINTS (NMP3-SL-2011-263104) and SUNFLOWER (FP7-ICT-2011-7, Grant No. 287594), the Swedish Research Council project grant No. 2013-4022 and the Göran Gustafsson Foundation for Research in Natural Sciences and Medicine.

\section{References}

[1] L. Hung, C. Tang, M. G. Mason, Appl. Phys. Lett. 1997, 70, 152.

[2] Y. D. Jin, X. B. Ding, J. Reynaert, V. I. Arkhipov, G. Borghs, P. L. Heremans, M. Van der Auweraer, Org. Electron. 2004, 5, 271.

[3] C. J. Brabec, S. E. Shaheen, C. Winder, N. S. Sariciftci, P. Denk, Appl. Phys. Lett. 2002, 80, 1288.

[4] V. D. Mihailetchi, J. K. J. van Duren, P. W. M. Blom, J. C. Hummelen, R. a. J. Janssen, J. M. Kroon, M. T. Rispens, W. J. H. Verhees, M. M. Wienk, Adv. Funct. Mater. 2003, $13,43$.

[5] S. Hoshino, S. Nagamatsu, M. Chikamatsu, M. Misaki, Y. Yoshida, N. Tanigaki, K. Yase, Jpn. J. Appl. Phys. 2002, 41, L808.

[6] A. J. Drew, J. Hoppler, L. Schulz, F. L. Pratt, P. Desai, P. Shakya, T. Kreouzis, W. P. Gillin, A. Suter, N. a Morley, V. K. Malik, A. Dubroka, K. W. Kim, H. Bouyanfif, F. Bourqui, C. Bernhard, R. Scheuermann, G. J. Nieuwenhuys, T. Prokscha, E. Morenzoni, Nat. Mater. 2009, 8, 109.

[7] L. S. Hung, R. Q. Zhang, P. He, G. Mason, J. Phys. D. Appl. Phys. 2002, 35, 103. 


\section{WILEY-VCH}

[8] X. Yang, Y. Mo, W. Yang, G. Yu, Y. Cao, Appl. Phys. Lett. 2001, 79, 563.

[9] S. E. Shaheen, G. E. Jabbour, M. M. Morrell, Y. Kawabe, B. Kippelen, N. Peyghambarian, M.-F. Nabor, R. Schlaf, E. a. Mash, N. R. Armstrong, J. Appl. Phys. 1998, 84, 2324.

[10] P. Piromreun, H. Oh, Y. Shen, G. G. Malliaras, J. C. Scott, P. J. Brock, Appl. Phys. Lett. 2000, 77, 2403.

[11] C. J. Huang, D. Grozea, a. Turak, Z. H. Lu, Appl. Phys. Lett. 2005, 86, 033107.

[12] M. Stößel, J. Staudigel, F. Steuber, J. Blässing, J. Simmerer, A. Winnacker, H. Neuner, D. Metzdorf, H.-H. Johannes, W. Kowalsky, Synth. Met. 2000, 111-112, 19.

[13] X. J. Wang, J. M. Zhao, Y. C. Zhou, X. Z. Wang, S. T. Zhang, Y. Q. Zhan, Z. Xu, H. J. Ding, G. Y. Zhong, H. Z. Shi, Z. H. Xiong, Y. Liu, Z. J. Wang, E. G. Obbard, X. M. Ding, W. Huang, X. Y. Hou, J. Appl. Phys. 2004, 95, 3828.

[14] Z. Sun, B. Ding, B. Wu, Y. You, X. Ding, X. Hou, J. Phys. Chem. C 2012, 116, 2543.

[15] Z. Sun, X. Ding, B. Ding, X. Gao, Y. Hu, X. Chen, Y. He, X. Hou, Org. Electron. 2013, $14,511$.

[16] X. Z. Wang, Z. T. Xie, X. J. Wang, Y. C. Zhou, W. H. Zhang, X. M. Ding, X. Y. Hou, Appl. Surf. Sci. 2007, 253, 3930.

[17] S. T. Zhang, X. M. Ding, J. M. Zhao, H. Z. Shi, J. He, Z. H. Xiong, H. J. Ding, E. G. Obbard, Y. Q. Zhan, W. Huang, X. Y. Hou, Appl. Phys. Lett. 2004, 84, 425.

[18] H.-J. Li, R.-H. Zhu, X.-Y. Li, Z.-J. Wang, B.-C. Yang, Solid State Commun. 2007, 144, 445.

[19] H. Ishii, K. Sugiyama, E. Ito, K. Seki, Adv. Mater. 1999, 11, 605.

[20] S. Braun, W. R. Salaneck, M. Fahlman, Adv. Mater. 2009, 21, 1450.

[21] S. Sanvito, Nat. Phys. 2010, 6, 562.

[22] P. Ruden, Nat. Mater. 2011, 10, 8.

[23] Y. Zhan, M. Fahlman, J. Polym. Sci. Part B Polym. Phys. 2012, 50, 1453.

[24] L. Schulz, L. Nuccio, M. Willis, P. Desai, P. Shakya, T. Kreouzis, V. K. Malik, C. Bernhard, F. L. Pratt, N. a Morley, A. Suter, G. J. Nieuwenhuys, T. Prokscha, E. Morenzoni, W. P. Gillin, a J. Drew, Nat. Mater. 2011, 10, 39.

[25] D. Ciudad, M. Gobbi, C. J. Kinane, M. Eich, J. S. Moodera, L. E. Hueso, Adv. Mater. 2014, 26, 7561 .

[26] T. D. Nguyen, E. Ehrenfreund, Z. V. Vardeny, Science 2012, 337, 204. 


\section{WILEY-VCH}

[27] M. Fahlman, P. Sehati, W. Osikowicz, S. Braun, M. P. de Jong, G. Brocks, J. Electron Spectros. Relat. Phenomena 2013, 190, 33.

[28] V. Dediu, L. Hueso, I. Bergenti, A. Riminucci, F. Borgatti, P. Graziosi, C. Newby, F. Casoli, M. De Jong, C. Taliani, Y. Zhan, Phys. Rev. B 2008, 78, 115203.

[29] M. Gobbi, F. Golmar, R. Llopis, F. Casanova, L. E. Hueso, Adv. Mater. 2011, 23, 1609.

[30] V. A. Dediu, L. E. Hueso, I. Bergenti, C. Taliani, Nat. Mater. 2009, 8, 707.

[31] L. Lindell, D. Çakır, G. Brocks, M. Fahlman, S. Braun, Appl. Phys. Lett. 2013, 102, 223301.

[32] K. Sugi, H. Ishii, Y. Kimura, M. Niwano, E. Ito, Y. Washizu, N. Hayashi, Y. Ouchi, K. Seki, Thin Solid Films 2004, 464-465, 412.

[33] S. T. Lee, X. Y. Hou, M. G. Mason, C. W. Tang, Appl. Phys. Lett. 1998, 72, 1593.

[34] M. G. Mason, C. W. Tang, L.-S. Hung, P. Raychaudhuri, J. Madathil, D. J. Giesen, L. Yan, Q. T. Le, Y. Gao, S.-T. Lee, L. S. Liao, L. F. Cheng, W. R. Salaneck, D. a. dos Santos, J. L. Brédas, J. Appl. Phys. 2001, 89, 2756.

[35] A. Kahn, N. Koch, W. Gao, J. Polym. Sci. Part B Polym. Phys. 2003, 41, 2529.

[36] M. Knupfer, H. Peisert, T. Schwieger, Phys. Rev. B 2001, 65, 033204.

[37] R. Poole, J. Jenkin, J. Liesegang, R. Leckey, Phys. Rev. B 1975, 11, 5179.

[38] Y. Zhan, M. de Jong, F. Li, V. Dediu, M. Fahlman, W. Salaneck, Phys. Rev. B 2008, 78, 045208.

[39] S. J. Kang, Y. Yi, C. Y. Kim, S. W. Cho, M. Noh, K. Jeong, C. N. Whang, Synth. Met. 2006, 156, 32 .

[40] R. Lof, M. van Veenendaal, B. Koopmans, H. Jonkman, G. Sawatzky, Phys. Rev. Lett. 1992, 68, 3924.

[41] P. Benning, D. Poirier, T. Ohno, Y. Chen, M. Jost, F. Stepniak, G. Kroll, J. Weaver, J. Fure, R. Smalley, Phys. Rev. B 1992, 45, 6899.

[42] S. Prada, U. Martinez, G. Pacchioni, Phys. Rev. B 2008, 78, 235423.

[43] N. J. Watkins, Y. Gao, J. Appl. Phys. 2003, 94, 1289.

[44] G. Greczynski, M. Fahlman, W. R. Salaneck, J. Chem. Phys. 2000, 113, 2407.

[45] S. K. M. Jönsson, W. R. Salaneck, M. Fahlman, J. Appl. Phys. 2005, 98, 014901.

[46] S. K. M. Jönsson, E. Carlegrim, F. Zhang, W. R. Salaneck, M. Fahlman, Jpn. J. Appl. Phys. 2005, 44, 3695. 


\section{WILEY-VCH}

[47] H. Tatewaki, Phys. Rev. B 1999, 60, 3777.

[48] M. Fahlman, a Crispin, X. Crispin, S. K. M. Henze, M. P. de Jong, W. Osikowicz, C. Tengstedt, W. R. Salaneck, J. Phys. Condens. Matter 2007, 19, 183202.

[49] W. Osikowicz, M. P. de Jong, W. R. Salaneck, Adv. Mater. 2007, 19, 4213.

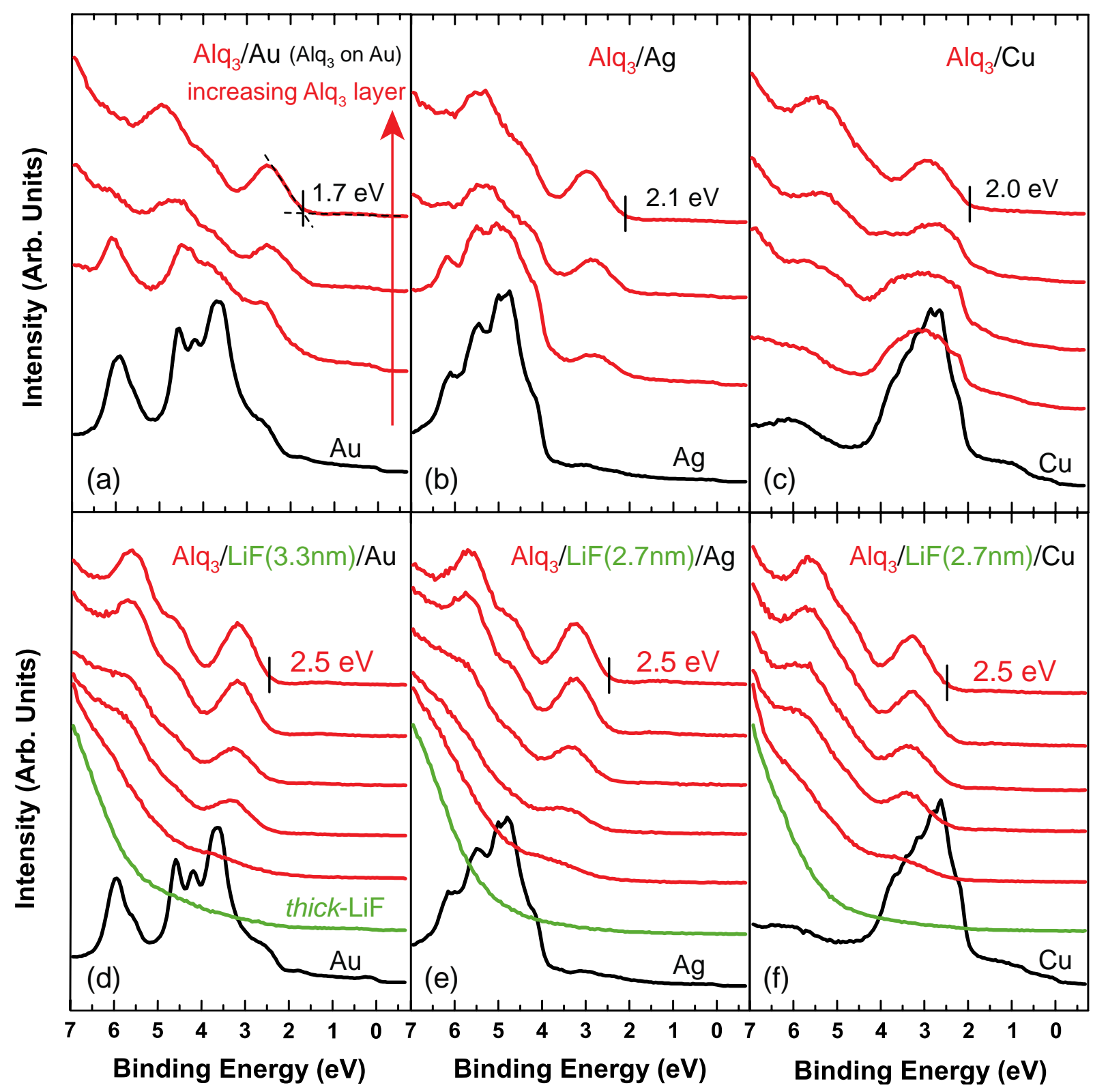

Figure 1. The UPS results of valence band of $\mathrm{Alq}_{3}$ on coinage metal with $3 \mathrm{~nm} \mathrm{LiF}$ interlayer and its reference without LiF: (a) gold, (b) silver, (c) copper, (d) LiF/gold, (e) LiF/silver and (f) $\mathrm{LiF} /$ copper. The spectra from bottom to top represent metal substrate, thick-LiF interlayer and stepwise deposited $\mathrm{Alq}_{3}$ layer in sequence, with black, green and red lines, respectively. 

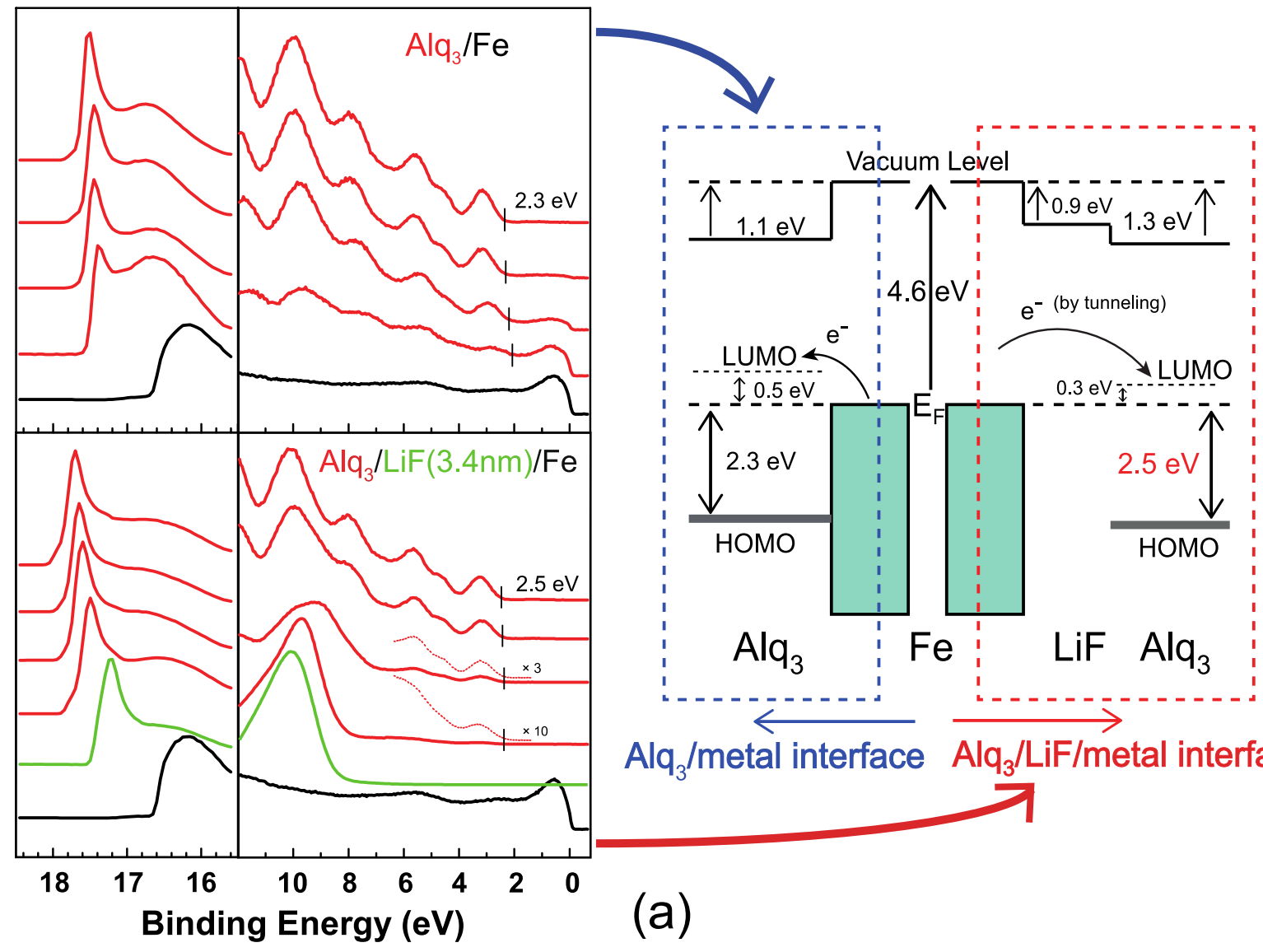

$\mathrm{Alq}_{3} /$ metal interface $\quad \mathrm{Alq}_{3} / \mathrm{LiF} /$ metal interface

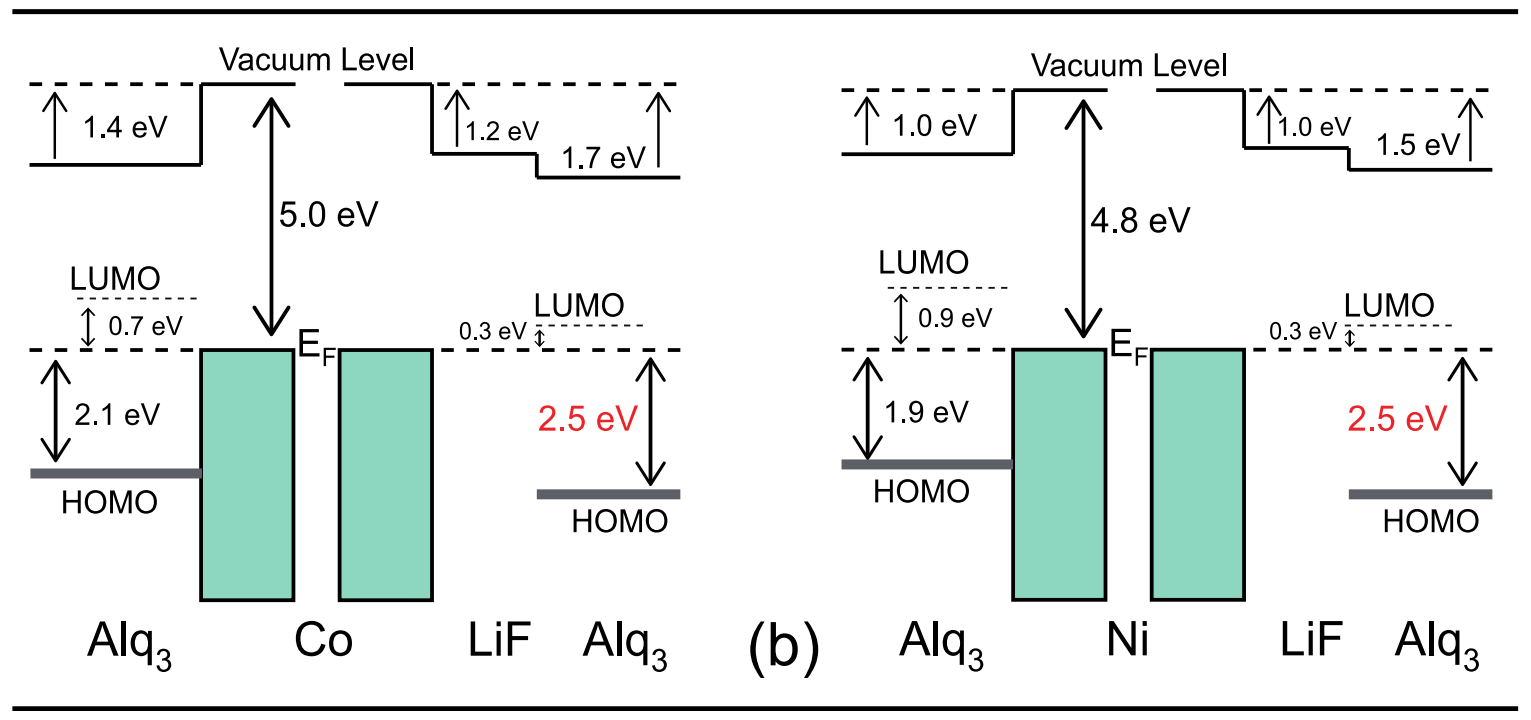

Figure 2. (a) Left: UPS results of secondary electron cut-off and valence band region of Alq on Fe substrate with $3 \mathrm{~nm} \mathrm{LiF}$ interlayer and its reference without LiF. Right: the comparison diagram of the energy level alignment derived from the left spectra; the left part corresponds to the case of $\mathrm{Alq}_{3} /$ metal interface while the right side to the case of $\mathrm{Alq}_{3} / \mathrm{LiF} / \mathrm{metal}$ interface. (b) The schematic diagram of the energy level alignment of the case of Co substrate and $\mathrm{Ni}$ substrate. 


\section{WILEY-VCH}
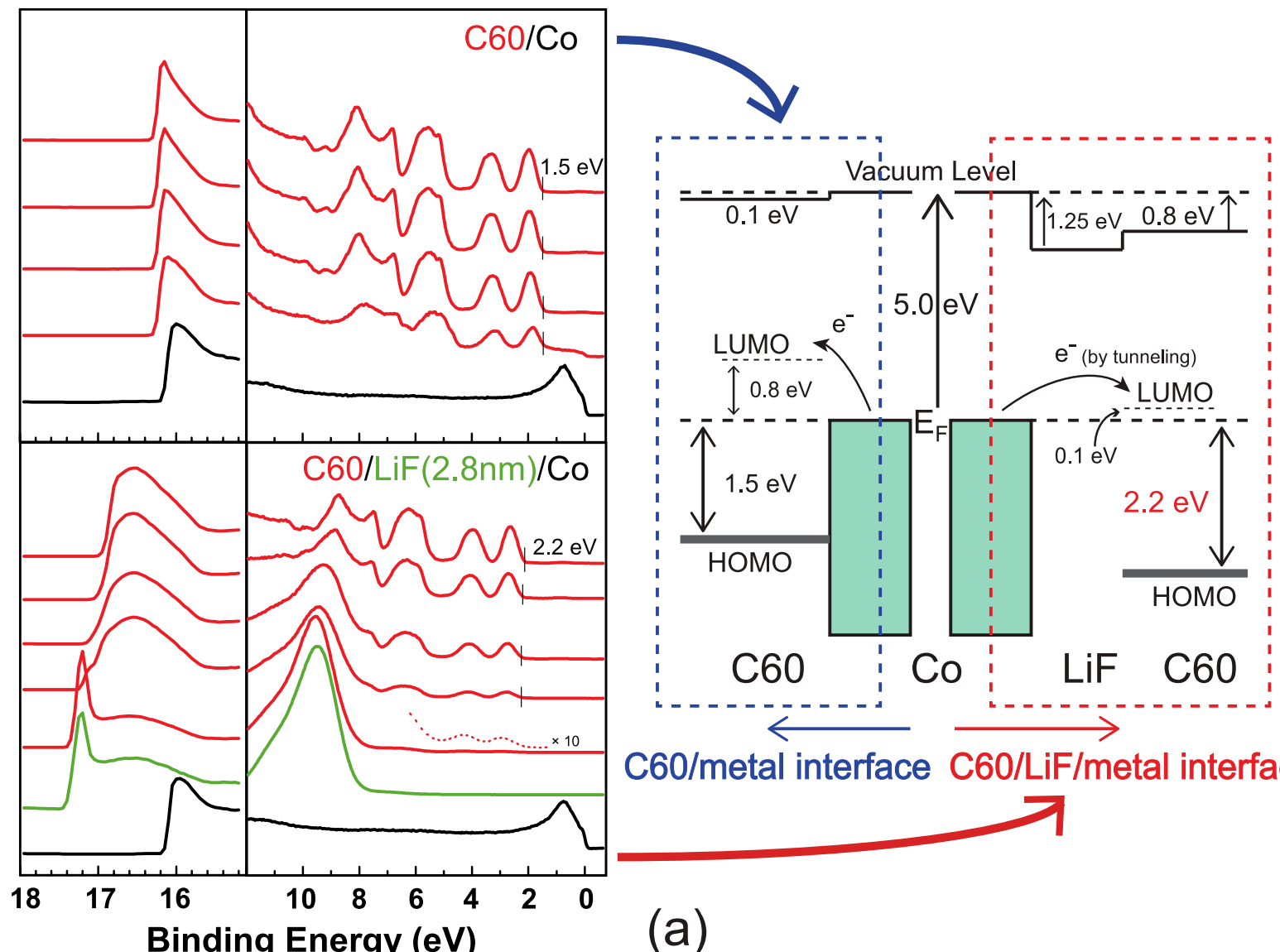

C60/metal interface C60/LiF/metal interface

(a)
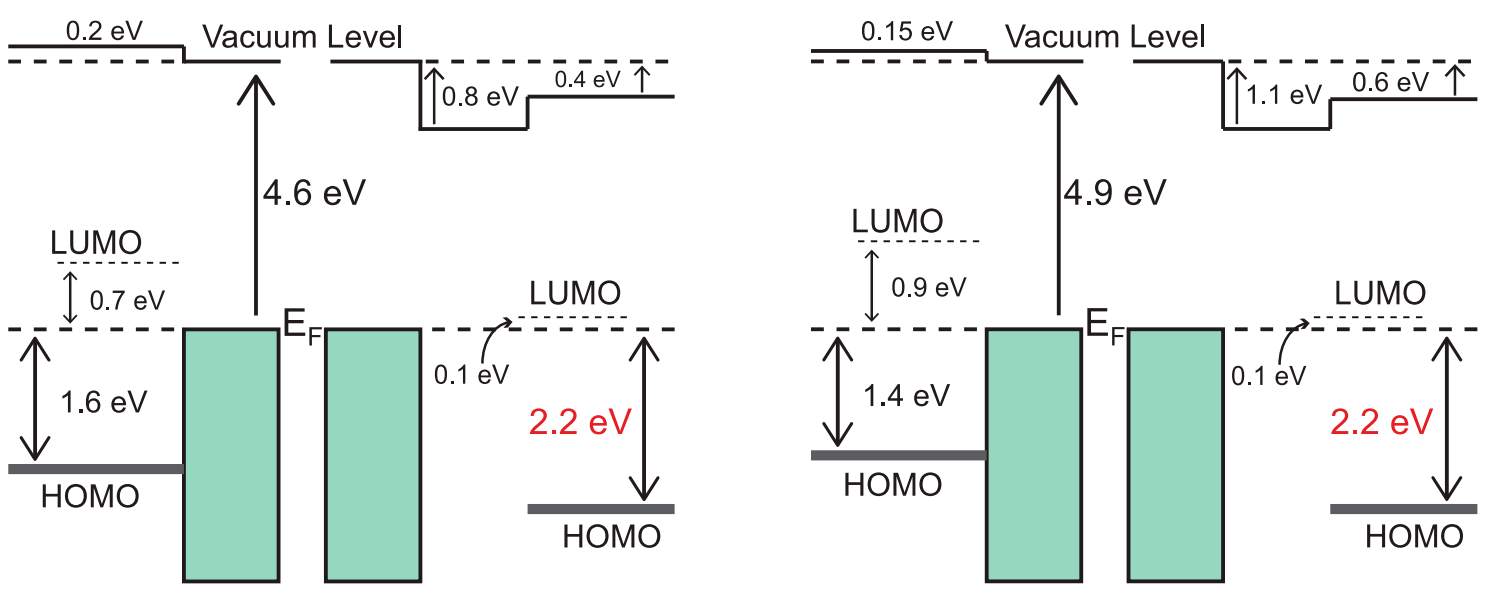

$\mathrm{C} 60$

$\mathrm{Fe}$

LiF $\quad$ C60

(b) $\mathrm{C} 60$

$\mathrm{Ni}$

LiF $\quad$ C60

Figure 3. (a) UPS results and the corresponding energy level alignment of C60 on Co substrate with and without $3 \mathrm{~nm} \mathrm{LiF}$ interlayer. (b) The schematic diagram of the energy level alignment of the case of $\mathrm{Fe}$ substrate and Ni substrate. 


\section{WILEY-VCH}

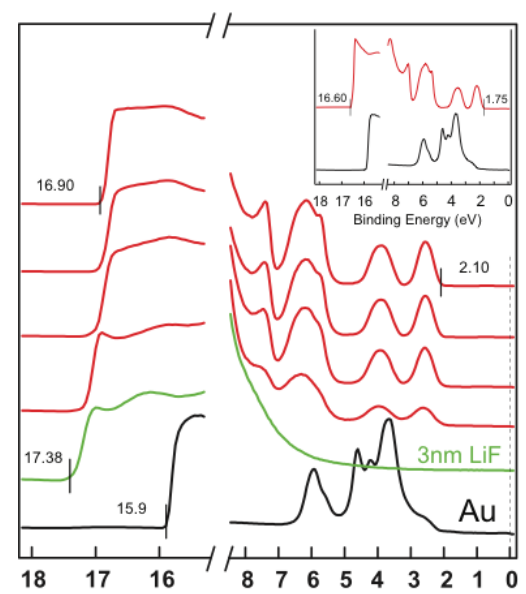

Binding Energy (eV)
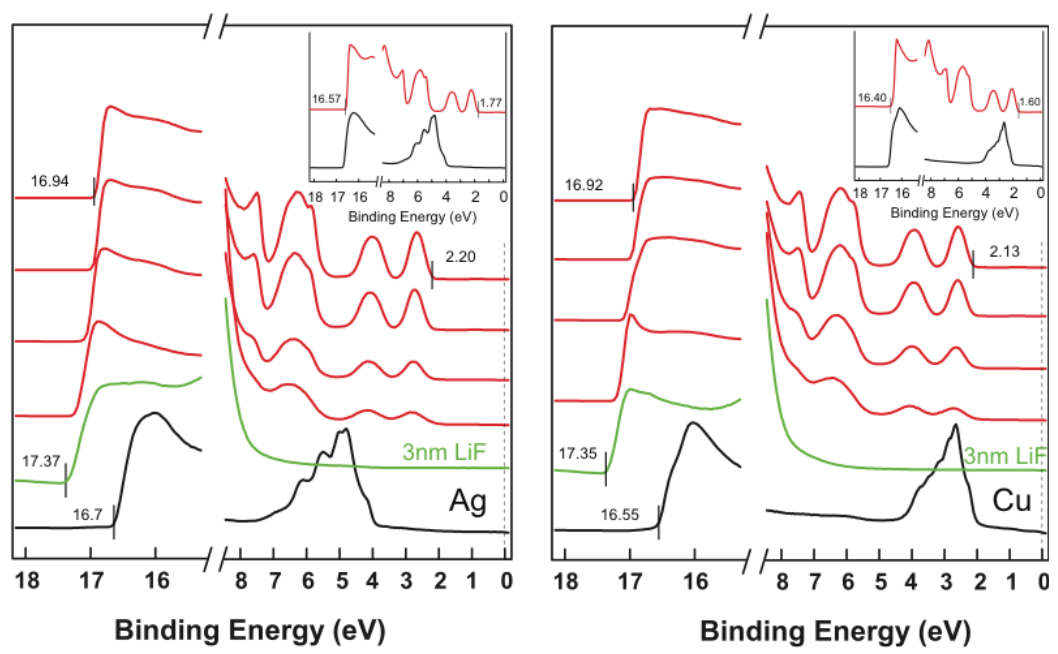

Figure 4. The UPS spectra at the interfaces of C60/coinage metals with the thick-LiF interlayer. The insets show the reference results of $\mathrm{C} 60$ on the coinage metals without LiF. 
(a)

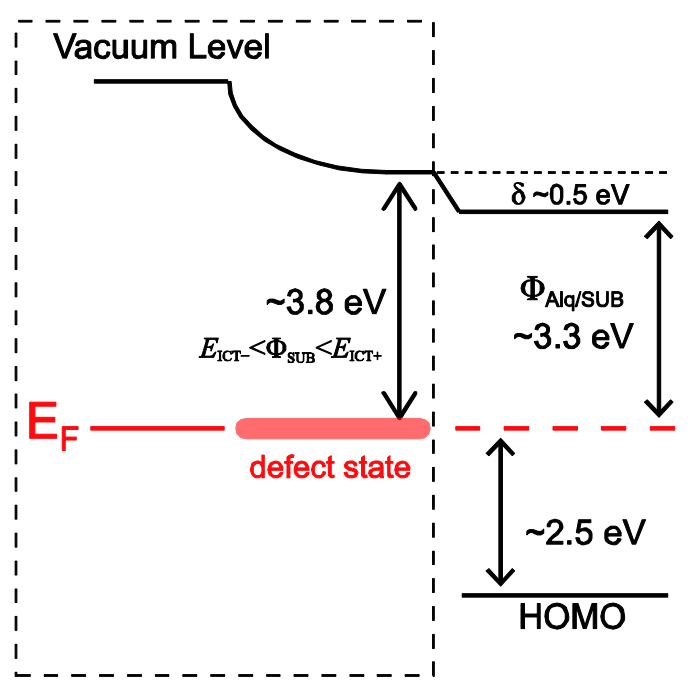

metal/thick LiF $\quad \mathrm{Alq}_{3}$

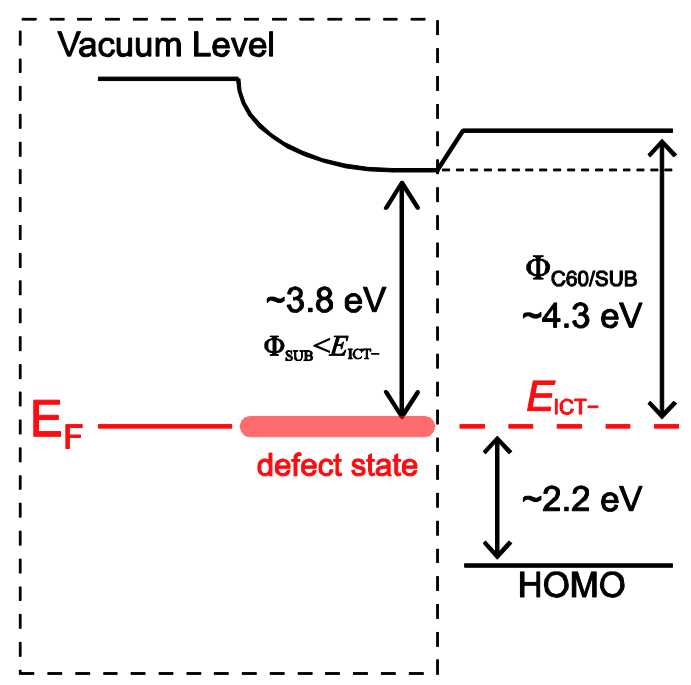

metal/thick LiF C60

(b)

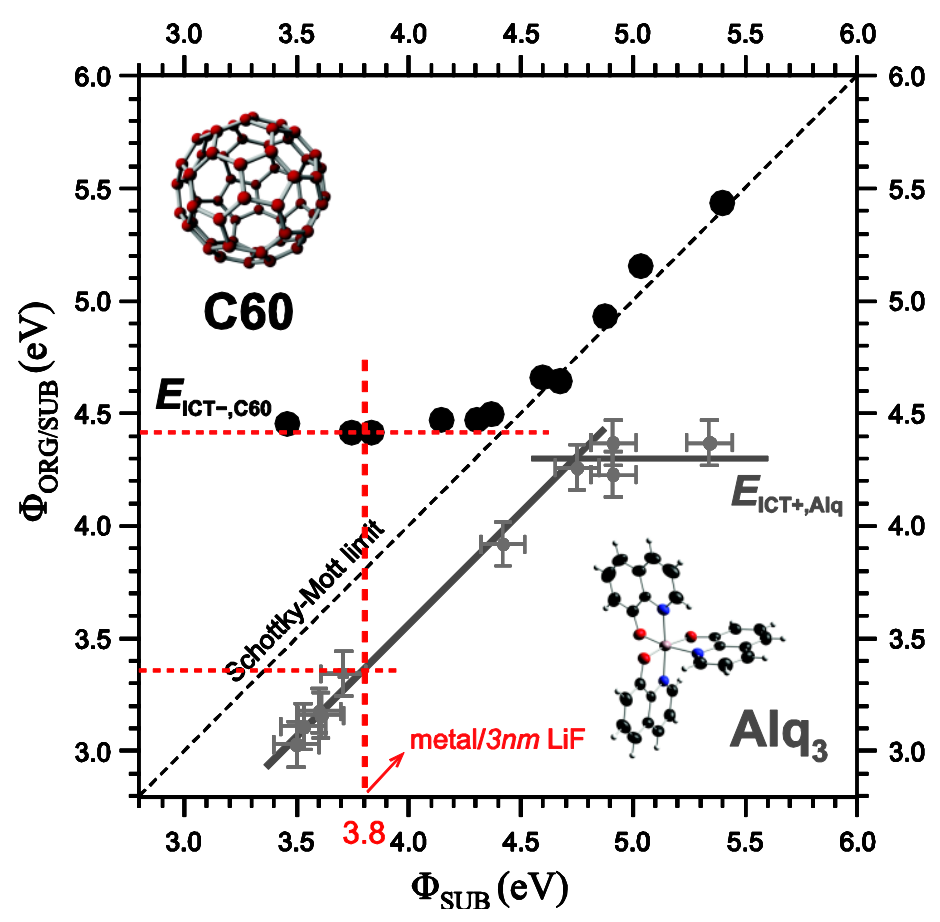

Figure 5. (a) LiF-assisted energy level pinning regime. The combination of metal and thick$\mathrm{LiF}$ forms a composite substrate, denoted by the dotted box. The energy level alignment of the organic material deposited on the new substrate follows the ICT model. (b) Dependence of $\Phi_{\mathrm{ORG} / \mathrm{SUB}}$ on $\Phi_{\mathrm{SUB}}$ for $\mathrm{Alq}_{3}$ and $\mathrm{C} 60$ that features typical ICT behavior (data taken from Refs. [31] and [49]). $E_{\mathrm{ICT}+} / E_{\mathrm{ICT}-}$ is the energy of the positive/negative ICT state; $\Phi_{\mathrm{SUB}}$ and $\Phi_{\mathrm{ORG} / \mathrm{SUB}}$ refer to the WF of substrate and organic-on-substrate. 